\title{
Réseaux de réactions dans l'oxydation catalytique de composés organiques: utilité et limitations
}

\author{
H.G. Lintz ${ }^{1}$ \\ 1 Laboratoire des sciences du génie chimique, Groupe ENSIC, 1, rue Grandville, 54001 Nancy Cedex - France \\ 1 Institut für Chemische Verfahrenstechnik, Universität Karlsruhe, D76128 Karlsruhe - Allemagne \\ e-mail : h-g.lintz@ciw.uni-karlsruhe.de
}

\begin{abstract}
Résumé - Des réseaux de réactions - aussi détaillés que nécessaire mais aussi simples que possible sont utilisés pour quantifier des cinétiques réactionnelles complexes dans le but d'améliorer la conduite du réacteur et de garantir un dimensionnement fiable. L'approche est illustrée à l'aide d'exemples, à savoir :

- la postcombustion catalytique de composés organiques volatiles ;

- l'oxydation partielle de composés organiques.
\end{abstract}

\begin{abstract}
Reaction Networks in Catalytic Oxidation of Organic Compounds: Utility and Limitations - Reaction networks - as detailed as necessary but as simple as possible - are used to quantify complex reaction kinetics with the aim to improve reactor operation and to guarantee a reliable scale-up. The approach is illustrated with the aid of examples, that is:

- the catalytic postcombustion of volatile organic compounds;

- the partial oxidation of organic compounds.
\end{abstract}

\section{INTRODUCTION}

La sélectivité du déroulement de réactions est la grandeur la plus importante si différentes séries de produits stoechiométriquement indépendants sont générées à partir des réactifs initiaux. Cependant, elle n'est correctement spécifiée que si elle se réfère à un volume de contrôle bien défini, par exemple un réacteur à lit fixe en état stationnaire [1]. Dans ce cas, la sélectivité globale observée est une fonction du taux de conversion des réactifs et dépend de la configuration du réacteur et du débit du fluide. Si l'on veut quantifier les propriétés de la composante catalytiquement active indépendamment des caractéristiques du réacteur et de la forme du grain catalytique, il faut déterminer des paramètres intrinsèques. Ainsi, la sélectivité expérimentalement accessible représente la limite supérieure qui peut être atteinte dans un réacteur [2]. La connaissance des paramètres intrinsèques essentiels permet alors la prédiction de la composition de la phase gazeuse dans le système réactif à l'aide d'un modèle mathématique reliant l'écoulement dans le réacteur à un réseau de réactions stoechiométriquement indépendantes.

L'utilisation de tels réseaux - aussi détaillés que nécessaire mais aussi simples que possible - permet de modéliser le comportement du réacteur. De cette façon, l'efficacité de différents catalyseurs peut être comparée et il est possible d'évaluer les performances du réacteur en fonction de :

- la composition du flux gazeux à l'entrée;

- la température initiale et son évolution ultérieure ;

- la nature du catalyseur et une structuration éventuelle du lit catalytique. 
L'objectif est d'améliorer le rendement, d'assurer un fonctionnement sûr du réacteur et de permettre un changement d'échelle fiable.

L'approche sera illustrée par la suite par l'exemple de deux groupes de réactions :

- la combustion catalytique de composés organiques volatils, où l'apparition d'intermédiaires toxiques est à proscrire et la sélectivité de leur formation à minimiser ;

- l'oxydation partielle de composés organiques, où, au contraire, le rendement en intermédiaire et la sélectivité de sa formation sont à maximiser.

Ces résultats ont fait l'objet de descriptions détaillées dans de précédentes publications.

Cet article a pour but de faire une synthèse et de donner une vue d'ensemble de l'approche choisie ainsi que de son utilité.

\section{DISPOSITIF EXPÉRIMENTAL ET EXPLOITATION DES MESURES}

Les mesures cinétiques ont été effectuées à l'aide de réacteurs tubulaires à lit fixe. Le dispositif expérimental est représenté schématiquement sur la figure 1 . Le flux et la composition du mélange réactionnel entrant sont réglés à l'aide de débitmètres thermiques; dans le cas de composantes liquides, un dispositif de saturation-condensation est utilisé.

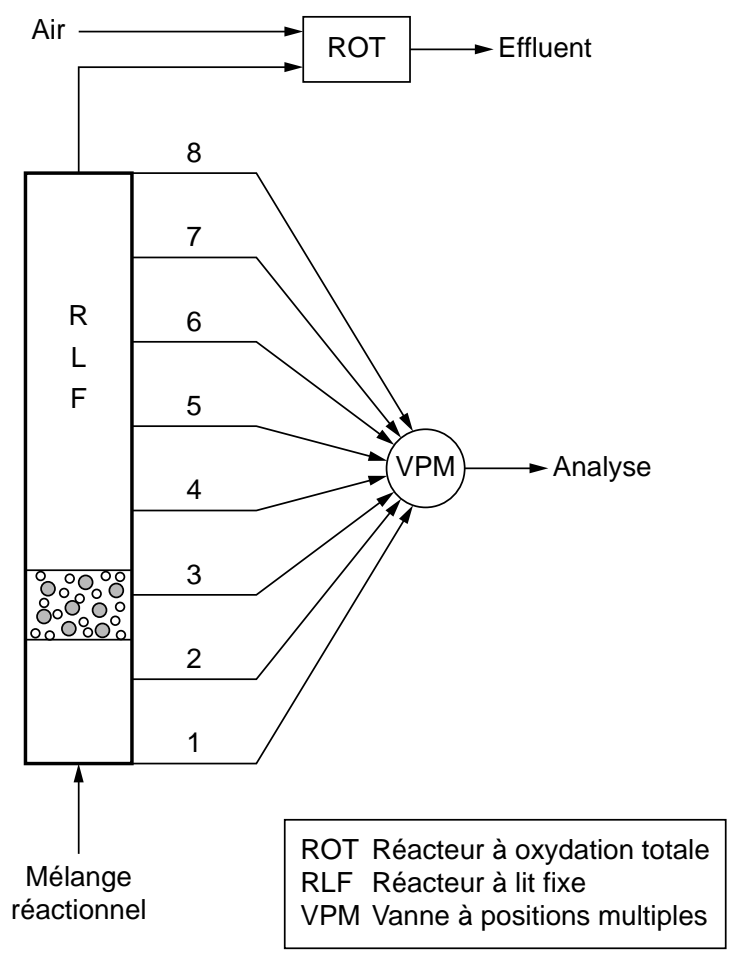

Figure 1

Schéma d'un réacteur à échantillonnage.

Schematic representation of the experimental set-up.
Au cœur de l'installation, le réacteur (longueur 1500 mm diamètre intérieur $15 \mathrm{~mm}$ ) est divisé en plusieurs segments de chauffage électrique séparés et de contrôle individuel de température afin de réaliser une opération isotherme. Des capillaires chauffés placés à l'entrée et à la sortie de chaque segment contiennent chacun un thermocouple supplémentaire et permettent de soutirer un faible débit de gaz. Une coupe de cette ouverture d'échantillonnage est représentée sur la figure 2. Grâce à ce dispositif, la composition de la phase gazeuse peut être déterminée le long du réacteur par utilisation d'une vanne à positions multiples qui dirige les différents échantillons successivement à la section analytique. Celle-ci comprend un chromatographe en phase gazeuse pour les composantes organiques, des spectrophotomètres à infrarouge non dispersifs pour les mesures de $\mathrm{CO}, \mathrm{CO}_{2}$ et éventuellement $\mathrm{H}_{2} \mathrm{O}$ ainsi qu'un instrument mécano-magnétique pour l'oxygène.

Le réacteur à échantillonnage est suivi d'un réacteur à oxydation totale (ROT) où tous les composés organiques sont transformés en $\mathrm{CO}_{2}$ et $\mathrm{H}_{2} \mathrm{O}$. L'échantillonnage à la sortie de ce deuxième réacteur et la mesure de la teneur en $\mathrm{CO}_{2}$ permettent la vérification du bilan de matière. La vérification du bilan de carbone donne des écarts inférieurs à $3 \%$.

Un exemple de paramètres opératoires lors d'une étude cinétique est donné dans le tableau 1. Il s'agit de l'oxydation partielle d'isobutène en méthacroléine sur un oxyde massique [3].

Le réacteur à échantillonnage est traité comme un système isotherme à écoulement piston.

Le bilan de matière entre l'entrée du réacteur et l'ouverture d'échantillonnage $z$ permet de caractériser l'évolution du mélange réactionnel le long du réacteur à l'aide de 3 grandeurs adimensionnelles. Il s'agit d'abord du taux de conversion de l'espèce $i$ en point $z$ :

$$
X_{i, z} \equiv \frac{\dot{n}_{i, o}-\dot{n}_{i, z}}{\dot{n}_{i, o}}
$$

et de la sélectivité de formation de l'espèce $j$ à partir de l'espèce $i$ :

$$
S_{i, j} \equiv \frac{\dot{n}_{j, z}}{\dot{n}_{i, o}-\dot{n}_{i, z}}
$$

où $\dot{n}_{i}$ désigne le flux molaire de l'espèce $i \mathrm{en} \mathrm{mol} \cdot \mathrm{s}^{-1}$.

La distribution des composantes carbonées est quantifiée à l'aide d'une concentration normalisée :

$$
y_{i, z} \equiv \frac{\dot{n}_{i, z} \cdot \varepsilon_{i}}{\dot{n}_{i, o} \cdot \varepsilon_{i}}
$$

où $\varepsilon_{i}$ désigne le nombre d'atomes de carbone contenus dans l'espèce $i$. Pour un produit $j, y_{j, z}$ est égal au rendement à l'endroit $z$. 


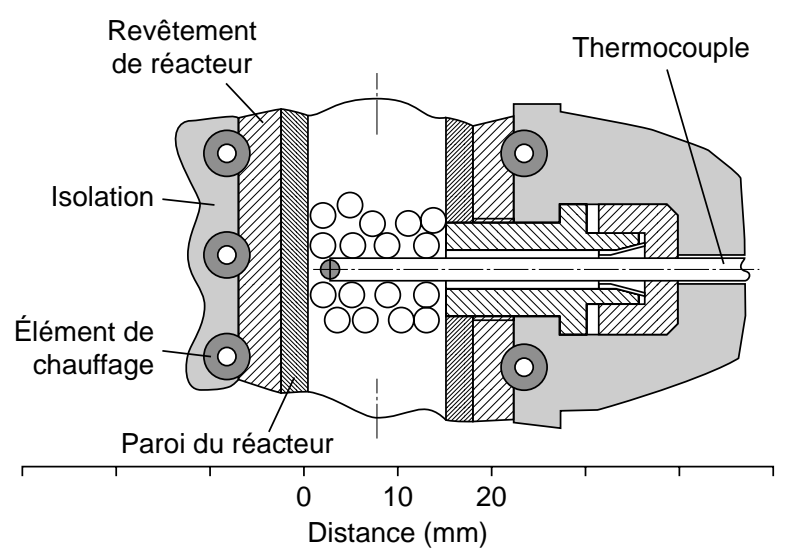

Figure 2

Prise d'échantillonnage.

Sampling port.

\section{TABLEAU 1}

Paramètres opératoires dans l'étude de l'oxydation catalytique d'isobutène

Operating parameters in the study of catalytic oxidation of isobutene

\begin{tabular}{l|l|l}
\hline Réacteur à échantillonnage & & \\
Température & $T_{R}$ & $=380 \ldots 420{ }^{\circ} \mathrm{C}$ \\
Pression d'entrée & $P$ & $=1,3 \mathrm{bar}$ \\
Masse du lit catalytique & $m_{k}$ & $=100 \ldots 200 \mathrm{~g}$ \\
Débit $\left(0^{\circ} \mathrm{C}, 1,013 \mathrm{bar}\right)$ & $V_{0}$ & $=20 \ldots 80 \mathrm{~cm}^{3} / \mathrm{s}$ \\
Fraction molaire initiale d'isobutène & $x_{\mathrm{iB}, 0}$ & $=3 \ldots 6 \%$ \\
Fraction molaire initiale d'oxygène & $x_{\mathrm{O}_{2}, 0}$ & $=10 \ldots 20 \%$ \\
Fraction molaire initiale d'eau & $x_{\mathrm{H}_{2} \mathrm{O}, 0}$ & $=0,10 \%$ \\
\hline Réacteur d'oxydation totale & & \\
Masse de catalyseur & $m_{\text {cat }}$ & $\approx 350 \mathrm{~g}$ \\
Température & $T$ & $=350 \ldots 550^{\circ} \mathrm{C}$ \\
Débit d'air supplémentaire & $\dot{V}_{\text {air,s }}$ & $=200 \mathrm{~cm}^{3} / \mathrm{s}$ \\
$\left(0{ }^{\circ} \mathrm{C}, 1,013\right.$ bar $)$ &
\end{tabular}

Un temps de séjour modifié est utilisé comme coordonnée spatiale :

$$
T_{m, z} \equiv \frac{m_{c, z}}{\dot{V}} ;\left[\tau_{m, z}\right]=\frac{g \cdot s}{m^{3}}
$$

où $m_{c, z}$ désigne la masse de la composante active du catalyseur contenue entre l'entrée et l'endroit $z$, et $\dot{V}$ désigne le flux volumique en $\mathrm{m}^{3} \cdot \mathrm{s}^{-1}$.

L'évolution des grandeurs adimensionnelles en fonction du temps de séjour guide le choix d'un réseau de réactions individuelles parallèles et consécutives. Le cas le plus simple a été décrit pour l'oxydation partielle du méthanol en formaldéhyde $[1,4]$.
Le réseau est constitué d'un schéma triangulaire :

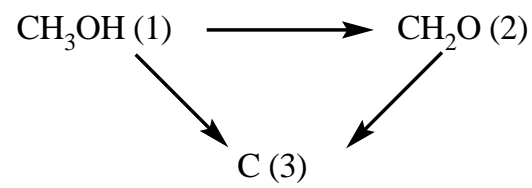

où $\mathrm{C}$ regroupe tous les produits carbonés secondaires. Dans le cas présent, les vitesses $r_{i j}$ de transformation sont du premier ordre par rapport au réactif et l'évolution du système peut être décrite à l'aide de trois coefficients cinétiques $k_{i j}$. De ce fait, le catalyseur peut être caractérisé par les paramètres suivants :

- l'activité dans la transformation du réactif:

$$
{ }^{G} k_{1}=k_{12}+k_{13}
$$

- la sélectivité de formation de l'intermédiaire :

$$
{ }^{G} S_{2}=\frac{k_{12}}{k_{12}+k_{13}}
$$

- la stabilité de l'intermédiaire :

$$
{ }_{G} \lambda_{2}=\frac{k_{12}}{k_{23}}
$$

Le symbole $G$ signale que le volume de contrôle considéré est le grain catalytique [1].

Un schéma triangulaire s'applique également dans l'oxydation partielle d'acroléine [5] et s'écrit dans ce cas :

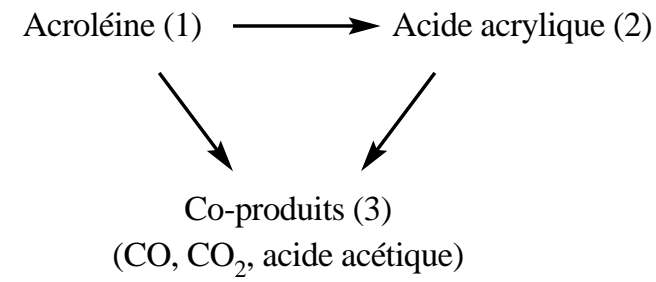

Il y a cependant une différence qui provient du fait que les réactions de transformation de l'acroléine sont d'ordre fractionnel par rapport à sa concentration. Afin de pouvoir utiliser quand même les paramètres définis pour les équations (57) pour caractériser et comparer différents catalyseurs, des constantes du pseudo-premier ordre peuvent être introduites comme cela a été décrit par exemple dans [6].

Le réseau de réactions et les expressions pour les vitesses de réaction mènent à un système d'équations différentielles. Il est résolu numériquement par la méthode de Runge-Kutta. L'ajustement des coefficients cinétiques est effectué à l'aide de l'algorithme Simplex de Nelder-Mead jusqu'au maximum de la fonction Likelyhood.

\section{RÉSULTATS}

La figure 3 représente des résultats typiques obtenus dans l'oxydation de l'acétone sur un catalyseur oxydique, $\mathrm{CuMn}_{2} \mathrm{O}_{4}$ [7]. On observe essentiellement trois produits, la concentration 


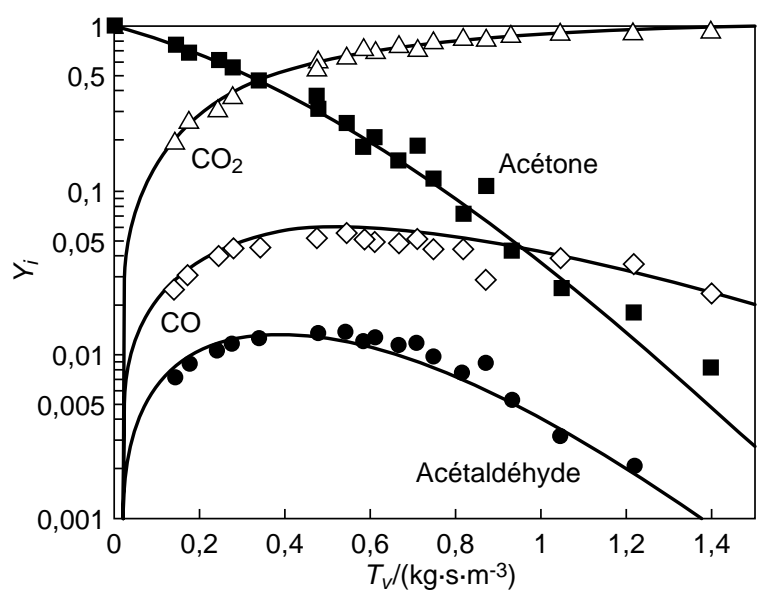

Figure 3

Concentration de réactifs en fonction du temps de séjour. Reactant concentration as a function of residence time. $x_{\text {acétone }, 0}=0,32 \% ; T_{R}=345^{\circ} \mathrm{C}$.

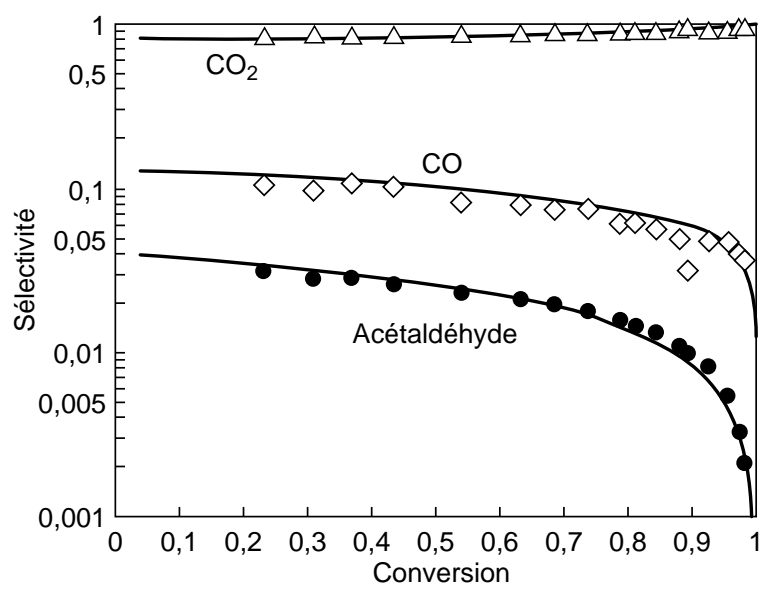

Figure 4

Sélectivités de réacteur en fonction du taux de conversion. Reactor selectivities as a function of conversion. $x_{\text {acétone, } 0}=0,32 \% ; T_{R}=345^{\circ} \mathrm{C}$. d'un quatrième, l'acide acétique, est d'un ordre de grandeur plus faible que celle de l'acétaldéhyde et il peut être négligé. La variation des sélectivités avec le taux de conversion est représentée sur la figure 4 . Nous constatons que les valeurs au taux de conversion égal à zéro, obtenues par extrapolation, sont non nulles. Ainsi, les trois produits sont formés simultanément à partir de l'acétone. Puisque seule la sélectivité de formation du $\mathrm{CO}_{2}$ augmente avec la conversion de l'acétone, le gaz carbonique peut être considéré comme le seul produit final de la réaction. L'examen des sélectivités nous amène donc à proposer le schéma réactionnel suivant :

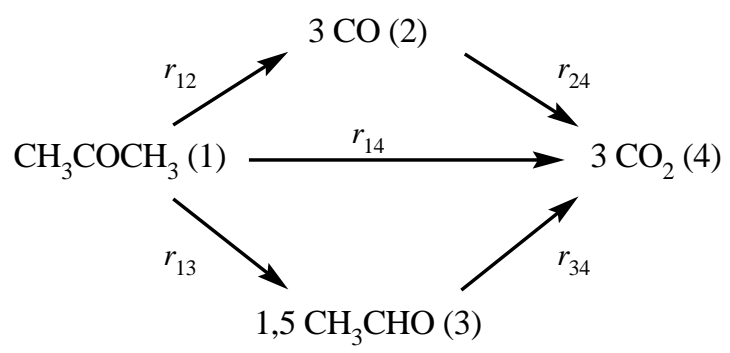

Il contient 5 étapes parallèles ou consécutives et peut être décrit à l'aide de 5 équations de vitesse. Il ressort des essais de modélisation que le schéma le plus simple se compose de 2 réactions du premier ordre et de 3 expressions contenant un coefficient supplémentaire au dénominateur :

$$
\begin{aligned}
& r_{12}=k_{12} C_{1} \\
& r_{13}=k_{13} C_{1}
\end{aligned}
$$

$$
\begin{aligned}
& r_{14}=\frac{k_{14} C_{1}}{1+b C_{1}} \\
& r_{24}=\frac{k_{24} C_{2}}{1+b C_{1}} \\
& r_{34}=\frac{k_{34} C_{3}}{1+b C_{1}}
\end{aligned}
$$

L'évolution du système réactionnel le long du réacteur se décrit ainsi à l'aide de 6 coefficients ajustés. L'accord entre l'expérience (points) et la modélisation (lignes) est satisfaisant, autant pour la variation des concentrations que pour celle des sélectivités. Dans l'intervalle de température étudié, la variation des coefficients cinétiques suit une relation du type Arrhenius.

Les figures 5 et 6 représentent des résultats analogues obtenus dans l'étude de l'oxydation partielle d'isobutène sur un catalyseur multicomposant $[3,6]$. Les produits de la réaction se composent de méthacroléine, d'acide méthacrylique et des produits d'oxydation ultérieure, essentiellement de $\mathrm{CO}$ et de $\mathrm{CO}_{2}$ mais aussi d'acide acétique, d'acétaldéhyde, d'acétone et d'acroléine dans des proportions inférieures à $1 \%$. Ils sont groupés dans une pseudo-espèce «produits d'oxydation ». L'examen des sélectivités (fig. 6) indique que seule le méthacroléine et les produits d'oxydation sont formés en parallèle à partir d'isobutène. L'acide méthacrylique est un descendant typique. Ceci amène au schéma suivant :

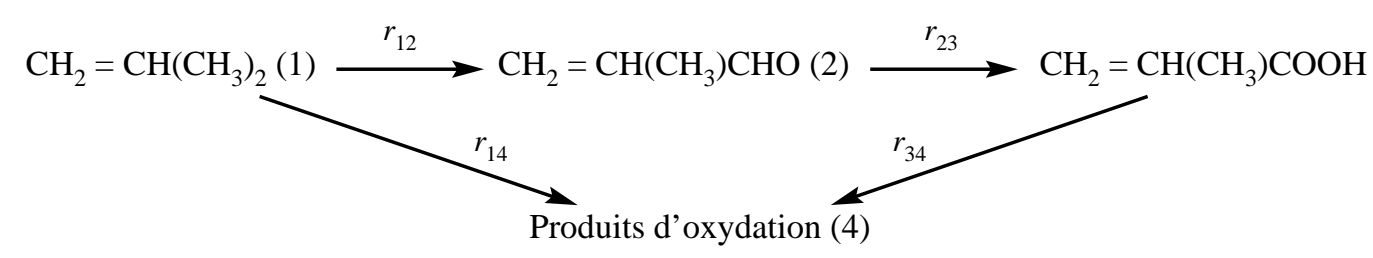




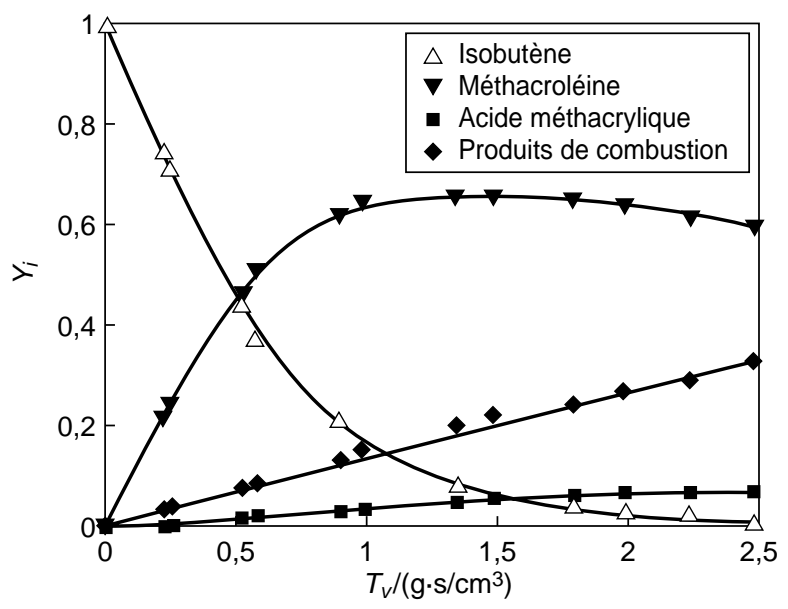

Figure 5

Concentration de réactifs en fonction du temps de séjour. Reactant concentration as a function of residence time.

$x_{\mathrm{C}_{4} \mathrm{H}_{8,0}}=5 \% ; x_{\mathrm{O}_{2}, 0}=20 \% ; T_{R}=400{ }^{\circ} \mathrm{C}$.

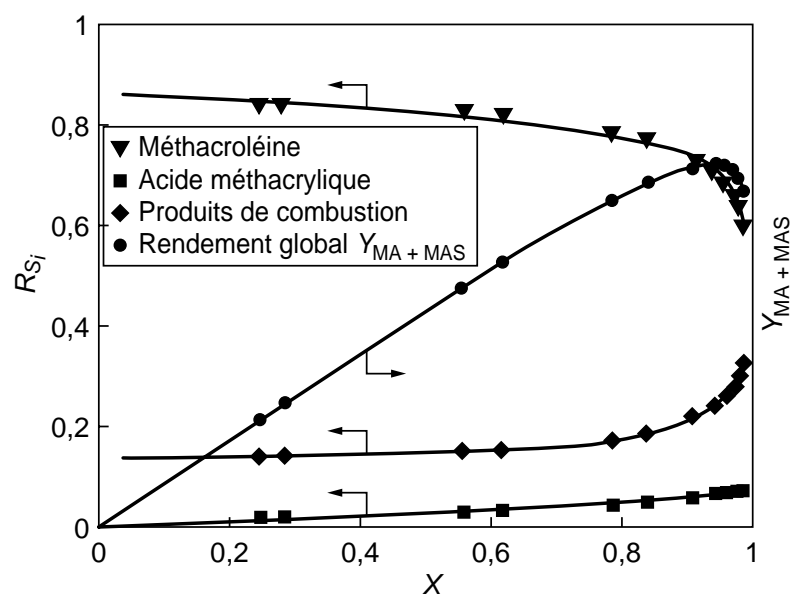

Figure 6

Sélectivité de réacteur en fonction du taux de conversion. Reactor selectivities as a function of conversion. $x_{\mathrm{C}_{4} \mathrm{H}_{8,0}}=5 \% ; x_{\mathrm{O}_{2}, 0}=20 \% ; T_{R}=400{ }^{\circ} \mathrm{C}$. car les essais de modélisation indiquent, de plus, que l'étape 24 peut être négligée.

Les réactions 23 et 34 sont du premier ordre, les étapes 12 et 14 sont décrites à l'aide d'un ordre fractionné, l'équation de vitesse contenant un terme supplémentaire au dénominateur, analogue aux équations (10-12). Les 5 coefficients cinétiques déterminés suivent une relation du type Arrhenius dans l'intervalle de température de 380 à $420^{\circ} \mathrm{C}$. Les figures 5 et 6 illustrent un bon accord entre l'expérience et la modélisation.

Dans l'étude citée [3, 6] l'effet de la composition du catalyseur a été étudié en détail. Celui-ci est composé de deux oxydes mixtes, tungstate de bismuth et molybdate de fer et de cobalt. Il est intéressant de noter que la forme des équations de vitesse reste la même dans tout le domaine de composition variant du tungstate pur en molybdate pur. L'effet synergique de la présence de deux solides distincts peut être quantifié à l'aide des paramètres définis au paragraphe précédent, c'està-dire l'activité du catalyseur, la sélectivité de formation et la stabilité de l'intermédiaire souhaité. Ceci est illustré dans la figure 7 où les valeurs de la sélectivité de formation de méthacroléine et sa stabilité sont tracées en fonction de la fraction massique du tungstate de bismuth. L'influence de la dimension et de la forme du catalyseur, de son mode de préparation et de la température de réaction peut être quantifiée de la même façon. L'étude a montré que la forme la plus adaptée du catalyseur consiste en cylindres creux (diamètre extérieur $4 \mathrm{~mm}$, diamètre intérieur $2 \mathrm{~mm}$, longueur $10 \mathrm{~mm}$ ) ce qui représente un bon compromis entre stabilité mécanique et efficacité du grain catalytique $\left(\eta \approx 0,9\right.$ à $420^{\circ} \mathrm{C}$, la température la plus élevée) [3]. La meilleure méthode de préparation est la précipitation du molybdate sur une poudre de tungstate à partir d'une solution aqueuse de précurseurs salins [6] .
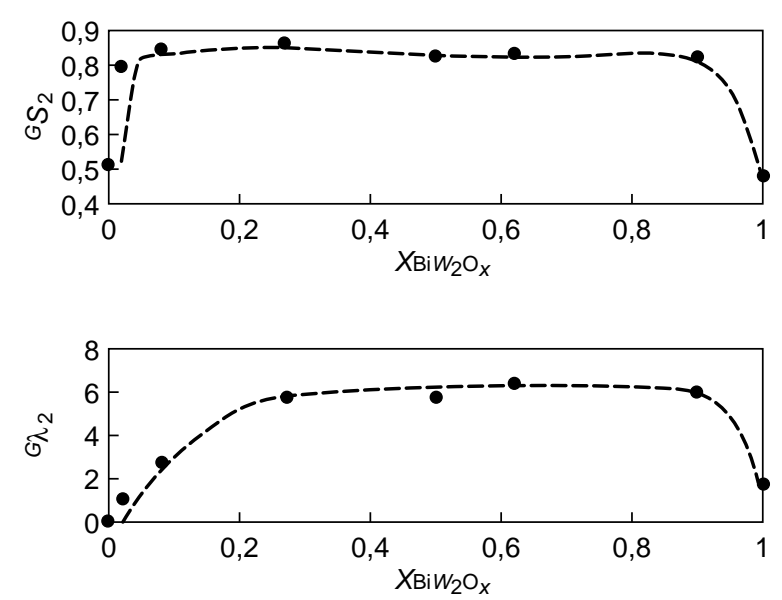

Figure 7

Sélectivité de formation et stabilité de méthacroléine en fonction de la composition du catalyseur exprimée en fraction massique de tungstate dans un mélange de tungstate et de molybdate.

Selectivity of formation and stability of methacrolein as a function of catalyst composition characterized by the mass fraction of tungstate in a mixture of tungstate and molybdate.

Il est surprenant que l'approximation de réseaux de réaction permette de décrire de façon satisfaisante l'évolution de concentration de diverses composantes sur plusieurs ordres de grandeur dans des systèmes réactionnels complexes. Tel est le cas dans l'oxydation d'isopropanol sur $\mathrm{CuMn}_{2} \mathrm{O}_{4}$. $\mathrm{La}$ figure 8 représente les valeurs expérimentales, le schéma ciaprès résume les étapes réactionnelles retenues. 


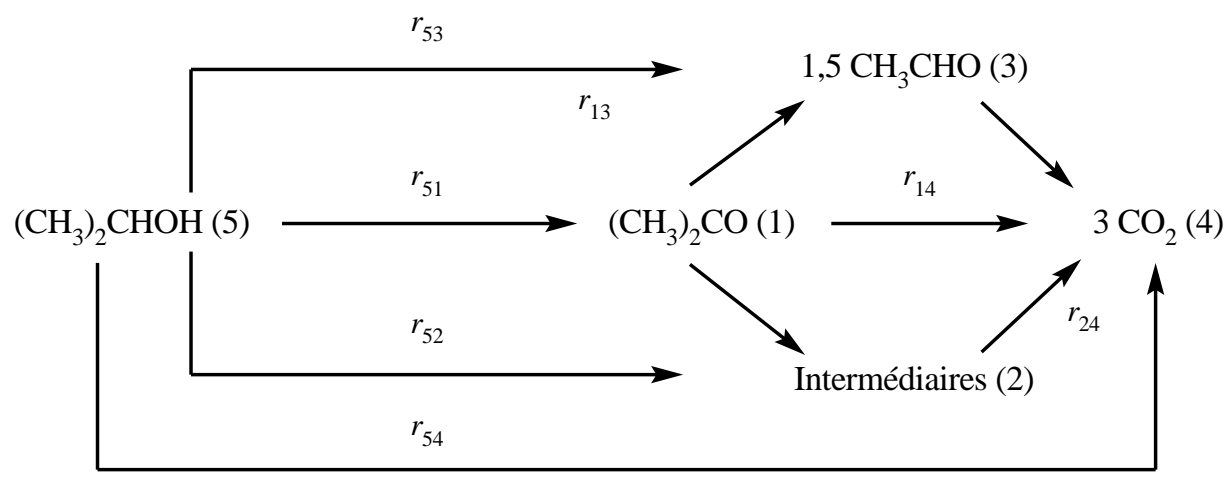

On remarque que le réseau de l'oxydation de l'acétone est inséré dans le schéma global où la pseudo-espèce «intermédiaires » regroupe essentiellement $\mathrm{CO}$, acide acétique et propène. Les coefficients cinétiques ajustés lors de l'étude de l'oxydation de l'acétone restent valables dans la modélisation globale à l'exception du terme $b$ au dénominateur. En effet, les expressions de vitesse de formation de $\mathrm{CO}_{2}$ ont maintenant la forme :

$$
r_{i 4}=\frac{k_{i 4} \cdot C_{i}}{1+b\left(C_{1}+C_{5}\right)}
$$

puisqu'il faut tenir compte des concentrations d'isopropanol et d'acétone, la valeur de $b$ est à ajuster à nouveau. Les courbes tracées sur la figure 8 illustrent le bon accord entre valeurs expérimentales et la modélisation.

L'approche «réseau » a été appliquée avec succès dans toute une série de réactions complexes. Elles sont résumées sur le tableau 2. En règle générale, un bon accord entre expériences et modélisation est obtenu après ajustement des coefficients cinétiques.

\section{DISCUSSION}

Dans le cas de la postcombustion catalytique de composantes organiques volatiles, l'intérêt primaire d'un réseau de réactions individuelles réside dans le fait qu'il permet de décrire et de prévoir l'évolution de la concentration d'intermédiaires toxiques. En effet, les limites légales pour de telles espèces ( $\mathrm{CO}$, aldéhydes, etc.) peuvent être nettement inférieures à celles du réactif initialement présent. Ceci est illustré par la figure 9 où l'évolution des concentrations de l'acétate de butyle et de l'acétaldéhyde est représentée conjointement avec les limites légales en RFA (TA-Luft). Nous observons que cette limite est atteinte dans l'oxydation du solvant à une

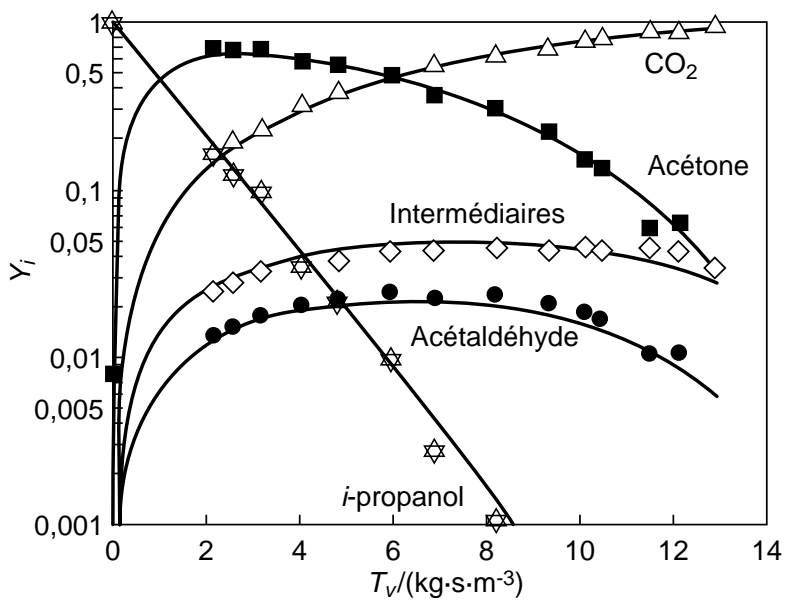

Figure 8

Concentration de réactif en fonction du temps de séjour. Reactant concentration as a function of residence time. $x_{i \text {-propanol,o }}=0,3 \% ; T_{R}=280^{\circ} \mathrm{C}$.

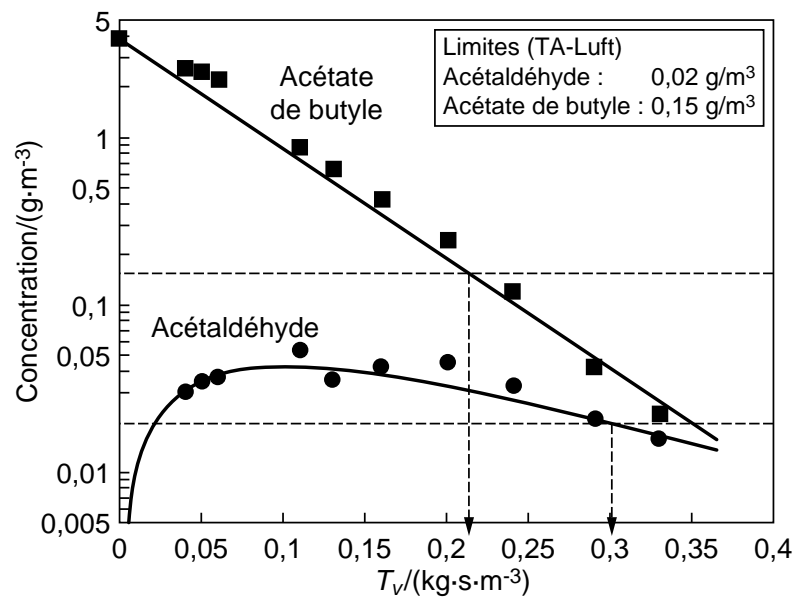

Figure 9

Oxydation d'acétate de butyle à $350^{\circ} \mathrm{C}$, concentrations massiques et limites d'émission.

Oxidation of butyle acetate at $350^{\circ} \mathrm{C}$, mass concentrations and emission limits. 
TABLEAU 2

Réseaux de reactions

Reaction networks

Oxydation partielle

\begin{tabular}{l|c|c|c}
\hline & Nombre de réactions & Coefficients & Références \\
\hline méthanol $\rightarrow$ formaldéhyde & 3 & 3 & {$[1,4]$} \\
$n$-butane $\rightarrow$ anhydride maléique & 3 & 3 & {$[8]$} \\
acroléine $\rightarrow$ acide acrylique & 3 & 4 & {$[5]$} \\
$i$-butène $\rightarrow$ méthacroléine (acide méthacrylique) & 4 & 5 & {$[6]$} \\
butadiène $\rightarrow$ furanne & 5 & 5 & {$[9]$} \\
propène $\rightarrow$ acroléine (acide acrylique) & 5 & 6 & {$[10]$} \\
$o$-xylène $\rightarrow$ anhydride phthalique & 6 & 7 & {$[11,12]$} \\
\hline
\end{tabular}

Ammoxydation

\begin{tabular}{c|c|c|c}
\hline & Nombre de réactions & Coefficients & Référence \\
\hline$p$-xylène $\rightarrow \begin{array}{c}p \text {-mononitrile } \\
p \text {-dinitrile }\end{array}$ & 5 & 5 & {$[13]$} \\
\hline
\end{tabular}

Postcombustion catalytique

\begin{tabular}{l|c|c|c|c}
\hline & Produits intermédiaires & Nombre de réactions & Coefficients & Référence \\
\hline Acétate de butyle & Acétaldéhyde, CO & 5 & 5 & \\
Acétone & Acétaldéhyde, CO & 5 & 6 & {$[14]$} \\
$i$-propanol & Acétone, acétaldéhyde, p.i. & 9 & 10 & \\
\hline
\end{tabular}

valeur du temps de séjour où elle est encore dépassée par l'intermédiaire toxique qui ne l'atteint qu'à une valeur du temps de séjour $50 \%$ plus élevée. Une telle prévision du comportement est essentielle dans la perspective d'utilisation de réacteurs équipés d'échangeurs thermiques régénérés [1517] comme le réacteur Matros à flux renversé [15] ou encore le réacteur Rotocat [18] construit en analogie avec l'échangeur rotatif du type Ljungstrom.
De même, l'utilisation d'un réseau de réactions peut éviter les pièges d'une approche trop simpliste qui consiste à ne déterminer que la température nécessaire à un taux de conversion suffisant pour atteindre la limite légale d'un composé organique. Ceci est illustré dans la figure 10 dans le cas de la postcombustion de l'acétone. Dans les conditions considérées, la limite légale est atteinte à $345^{\circ} \mathrm{C}$ avec un taux de conversion de $98 \%$ (partie gauche de la figure), le temps de
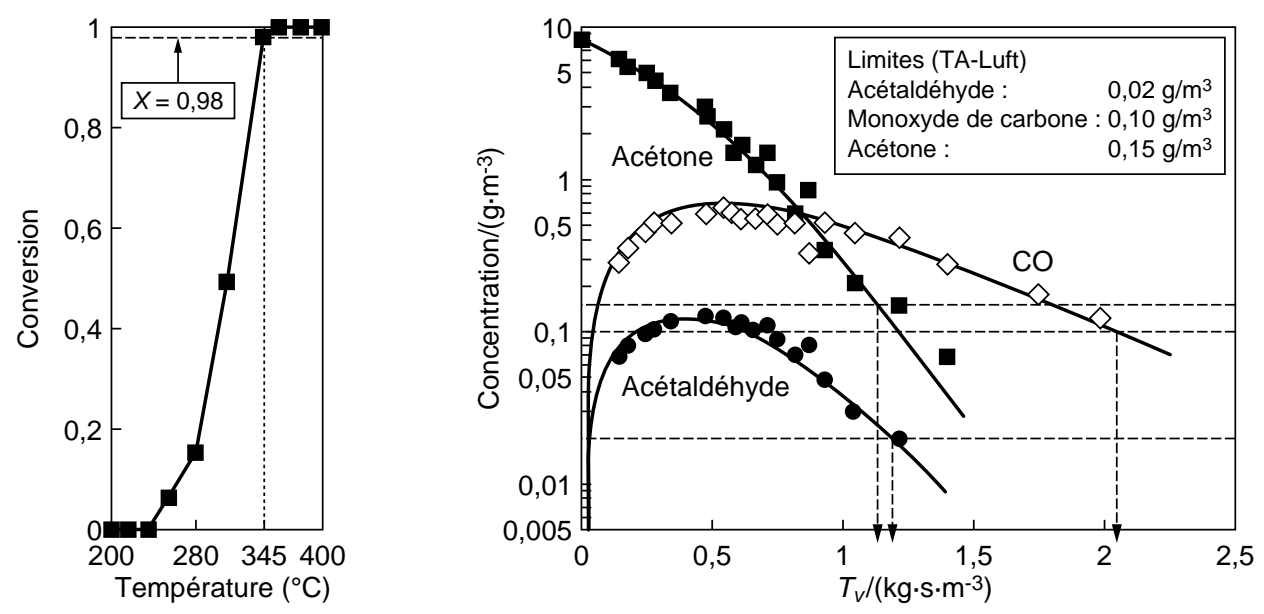

Figure 10

Oxydation de l'acétone, mesures d'orientation et profils de concentration.

Oxidation of acetone, screening measurements and concentration profiles. 
séjour étant égal à $1,1 \mathrm{~kg} \cdot \mathrm{s} \cdot \mathrm{m}^{-3}$. L'évolution des concentrations en fonction du temps de séjour, représentée sur la partie droite de la figure 10 , indique clairement qu'à cette température, la diminution de la concentration en $\mathrm{CO}$ reste largement insuffisante car il faudrait doubler le temps de séjour pour rester dans les limites légales.

Dans les procédés de postcombustion catalytique, l'excès d'air est considérable. De ce fait, il n'est pas surprenant qu'il ne faille pas tenir compte de la concentration d'oxygène dans l'expression des vitesses de réaction individuelle. Ceci n'est plus le cas dans l'oxydation partielle de composés organiques. En effet, le bon accord entre expérience et modélisation, illustré par exemple dans les figures 5 et 6 , est obtenu après l'ajustage des paramètres cinétiques dont les valeurs sont fonction des conditions initiales et doivent être réajustées si ces dernières sont modifiées [3].

Le problème a été discuté en détail [19] à l'exemple de l'oxydation partielle de butadiène. Dans ce cas le composé recherché est le furanne, d'autres produits intermédiaires comprennent l'acroléine, l'acétaldéhyde, l'acide acétique, le benzène, etc., et comme produits finaux figurent $\mathrm{CO}$ et $\mathrm{CO}_{2}$. Les résultats peuvent être modélisés à l'aide de 5 réactions individuelles du premier ordre par rapport aux composés organiques.

La figure 11 montre l'excellent accord entre l'expérience et la modélisation. Mais, cet accord peut être obtenu à l'aide de deux réseaux différents présentés dans le schéma ci-contre sans que la précision de la modélisation permette d'éliminer l'une ou l'autre possibilité sans informations supplémentaires.

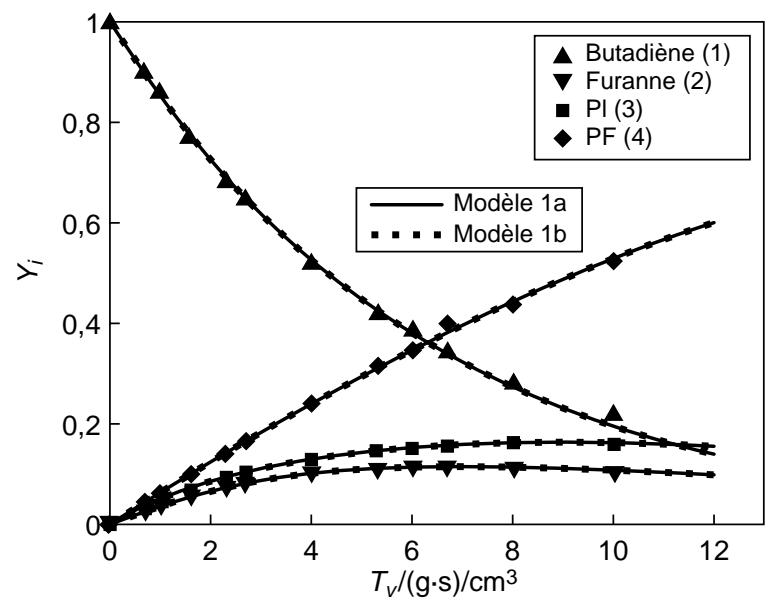

Figure 11

Oxydation partielle du butadiène - expérience et modélisation. $T_{R}=420^{\circ} \mathrm{C}$, catalyseur $\alpha / \gamma$ molybdate de bismuth. Composition à l'entrée du réacteur :

$x_{\mathrm{C}_{4} \mathrm{H}_{6}}=0,02, x_{\mathrm{O}_{2}}=0,07, x_{\mathrm{H}_{2} \mathrm{O}}=0,1$, reste $\mathrm{N}_{2}$.

Partial oxidation of butadiene - experiment and modelling $T_{R}=420^{\circ} \mathrm{C}$, catalyst $\alpha / \gamma$ bismuth molybdate composition at reaction inlet:

$x_{\mathrm{C}_{4} \mathrm{H}_{6}}=0.02, x_{\mathrm{O}_{2}}=0.07, x_{\mathrm{H}_{2} \mathrm{O}}=0.1$, balance $\mathrm{N}_{2}$.

\section{Modèle la}

Furanne

(2)

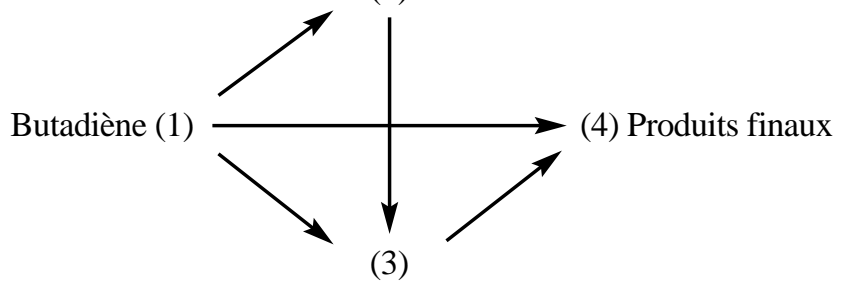

Produits

intermédiaires

\section{Modèle 16}

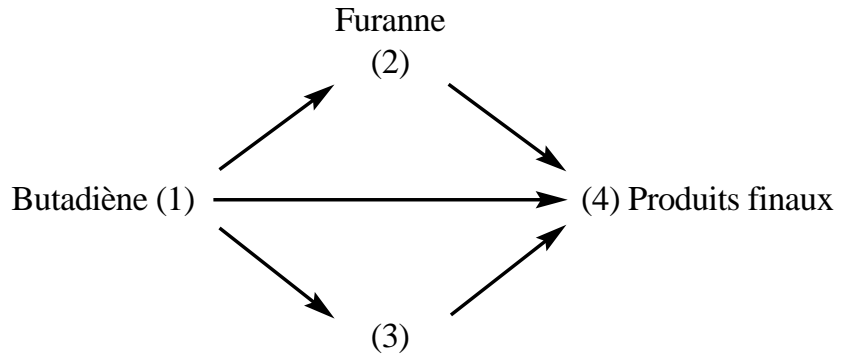

Produits

intermédiaires

Dans le cas présent, ce problème d'unicité n'est qu'apparent. En effet, le produit cherché étant le furanne, ni la sélectivité de sa formation ni la stabilité $\lambda$ ne sont affectées par les chemins de sa réaction ultérieure.

L'accord entre l'expérience et le calcul a été obtenu après ajustage de 5 coefficients cinétiques adaptés aux conditions initiales $(2 \%$ de butadiène et $7 \%$ d'oxygène à l'entrée du réacteur). Les valeurs ne permettent pas la simulation de l'expérience après une modification de ces valeurs initiales. Ceci est illustré sur la figure 12 où les résultats du calcul sont comparés aux expériences pour 3 compositions différentes à l'entrée du réacteur. Le modèle 1a, 5 réactions du premier ordre, ne permet pas une simulation généralisée.

Ceci devient possible si, dans une deuxième approximation (modèle II), les expressions des vitesses sont plus compliquées tout en gardant le même réseau (1a) de réactions couplées. Elles contiennent maintenant les valeurs initiales de la concentration de butadiène et tiennent compte de la variation de la concentration d'oxygène. Suivant la réaction individuelle, l'ordre par rapport à l'oxygène est de 0,4 ou de 0,5 . L'ordre est également fractionnel par rapport aux composés organiques et le système contient 6 coefficients cinétiques ajustables. Les résultats de la figure 12 montrent clairement qu'après ajustage, l'accord entre l'expérience et le calcul reste très bon si les conditions initiales sont modifiées.

Le modèle II a une validité plus générale que le modèle I aux dépens de sa simplicité. 


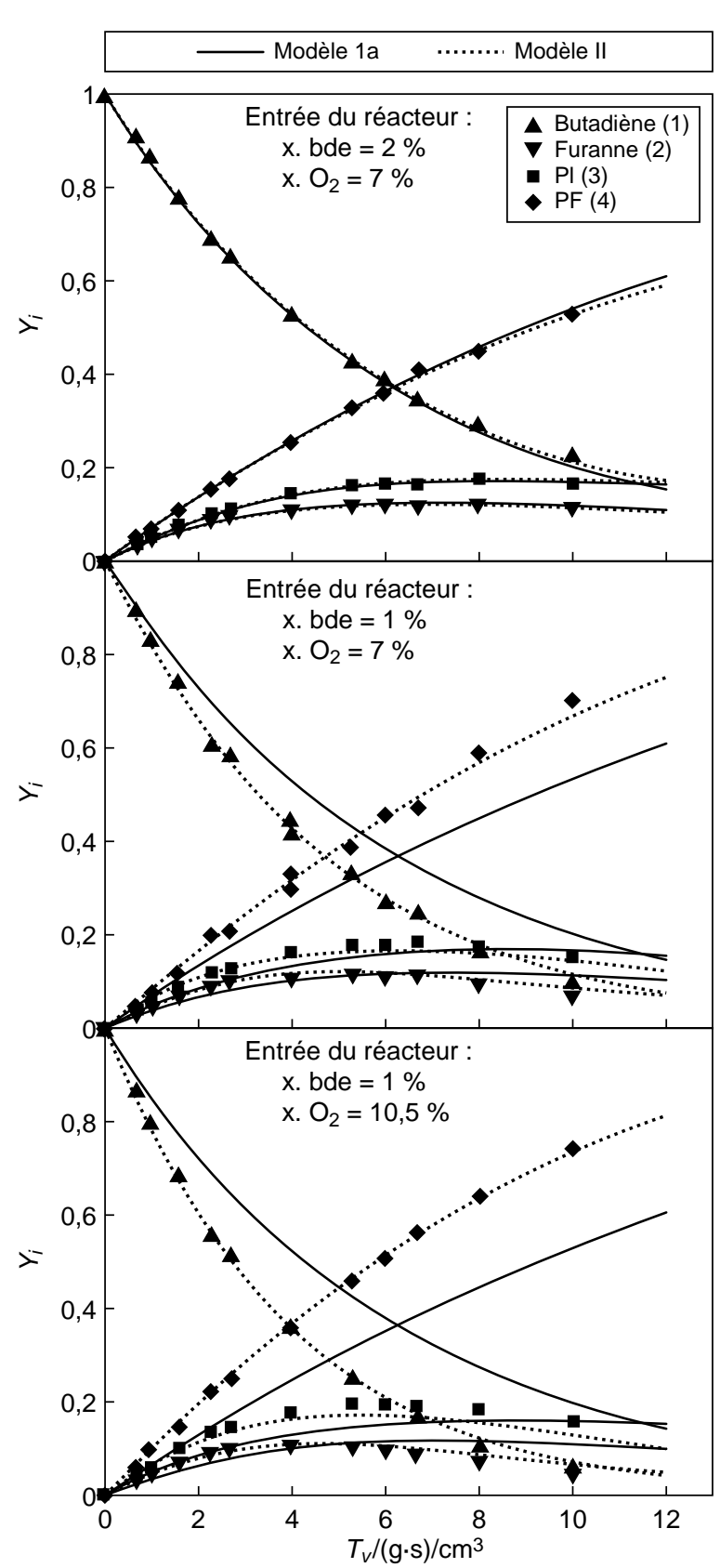

Figure 12

Valeurs expérimentales et résultats de simulation des modèles 1a et II, variation de la composition à l'entrée du réacteur $\left(x_{\mathrm{H}_{2} \mathrm{O}}=0,1\right) ; T_{R}=420^{\circ} \mathrm{C}$, catalyseur $\alpha / \gamma$ molybdate de bismuth.

Experimental values and results of fitting calculation for models $1 a$ and II, variation of feed gas composition $\left(x_{\mathrm{H}_{2} \mathrm{O}}=\right.$ $0.1) ; T_{R}=420^{\circ} \mathrm{C}$, catalyst $\alpha / \gamma$ bismuth molybdate.

Ainsi il est possible de généraliser la description par modèle en compliquant les expressions de vitesse de réaction. Il reste la question de savoir dans quel contexte cette complexité supérieure est justifiée.
La formulation du réseau de réaction représente tout d'abord l'évolution d'un système réactionnel complexe et donne une interprétation qualitative. Ensuite, un modèle quantitatif contenant la forme adaptée des vitesses de réaction, fournit un nombre limité de paramètres cinétiques après ajustement. Ceux-ci peuvent être utilisés pour caractériser la performance de catalyseurs différents sous des conditions variées, c'est-à-dire fournir certaines valeurs numériques qui décrivent de façon précise et complète le résultat d'une série de mesures. Ces valeurs numériques sont des paramètres ajustés ou des grandeurs dérivées de ceux-ci. La comparaison de telles valeurs guidera le choix du catalyseur et des conditions de son application. Cette comparaison n'a de sens que si les valeurs caractéristiques ressortent de modèles équivalents et si l'accord entre l'expérience et le calcul est de la même précision dans les cas considérés.

Dans le cas considéré, l'avantage du modèle I par rapport au modèle II vient du fait que :

- le traitement numérique est plus facile ;

- le nombre de paramètres ajustés est plus faible ;

- ce nombre limité et la forme plus simple des expressions de vitesse rendent les paramètres déduits $(S, \lambda)$ bien comparables.

Dans le cas présent, les paramètres déduits des coefficients de vitesse ont été utilisés pour comparer les performances catalytiques de différents molybdates de bismuth [19]. Nous allons observer plus loin qu'une forme simple et imparfaite du modèle n'empêche pas une extrapolation prudente et la simulation de l'influence de paramètres opérationnels afin d'améliorer la performance du réacteur.

Auparavant, il faut justifier que l'approche expérimentale est suffisante pour déduire un modèle basé sur des réseaux, car nous ne mesurons pas directement les vitesses de réaction mais l'évolution des concentrations en phase gazeuse le long du réacteur. C'est pourquoi les résultats ont été comparés avec des valeurs obtenues lors d'une étude cinétique détaillée en réacteur différentiel à recyclage [20] où la variation des vitesses de réaction en fonction de la concentration des réactifs est obtenue directement. Il a été démontré, à l'exemple de l'oxydation partielle de l'acroléine en acide acrylique, que les deux approches sont complémentaires, appliquées dans un but différent, et fournissent des résultats consistants. Le réacteur à recyclage est utilisé dans l'étude de réactions suffisamment simples pour élucider des détails de la séquence de réactions élémentaires et déterminer la cinétique fondamentale. L'approche intégrale en réacteur à échantillonnage est adaptée aux systèmes plus complexes dans le but de comparer les performances de catalyseurs et de prédire les variations de rendement et de sélectivité en fonction des conditions opératoires.

Cette dernière possibilité sera illustrée à l'exemple de l'oxydation partielle d'isobutène $[3,6]$. En effet, l'étude détaillée avait montré que la sélectivité de formation et la stabilité de l'intermédiaire acroléine variaient de façon opposée avec la température. 
L'augmentation de la sélectivité était accompagnée d'une diminution de la stabilité. De ce fait, il semblait probable qu'une structuration en température du réacteur améliore le rendement en produits partiellement oxydés si cette température était réduite à partir d'un certain taux de conversion de l'isobutène.

Ceci fut confirmé par la simulation et validé a posteriori par l'expérience. Les résultats sont présentés sur la figure 13 où l'opération isotherme est comparée à la mise en œuvre d'un lit structuré. Les courbes tracées correspondent au calcul et sont confirmées par les points expérimentaux. Ainsi, l'expérience valide les résultats de la simulation effectuée avec les valeurs ajustées des coefficients cinétiques, déterminées sous conditions isothermes pour la même composition gazeuse à l'entrée du réacteur. Dans le cas d'un lit fixe composé de deux catalyseurs différents ajoutés à la structuration en température ( 3 zones), le rendement en intermédiaire peut être augmenté encore [3].

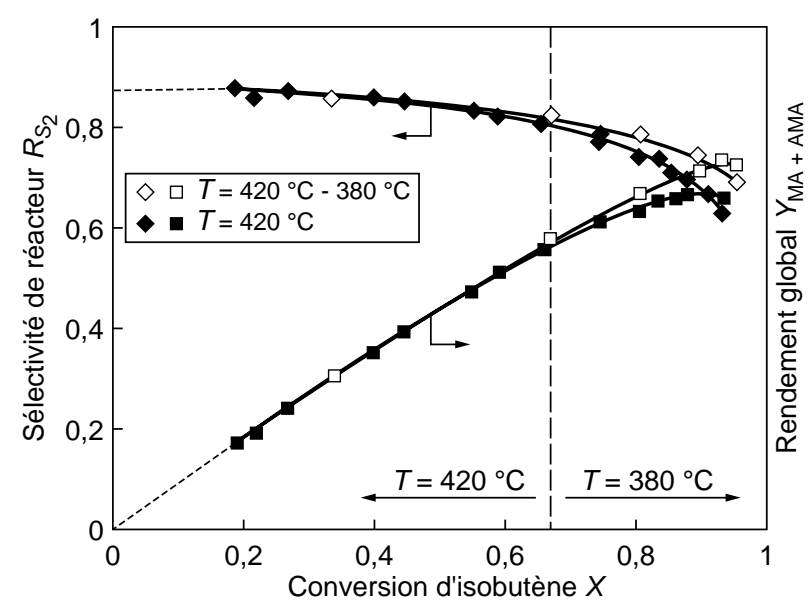

Figure 13

Sélectivité de la formation de méthacroléine et rendement global en fonction de la conversion d'isobutène sous conditions isothermes et dans un réacteur à 2 zones de température.

Reactor selectivity of methacrolein formation and global yield as a function of isobutene conversion at isothermal conditions and in a reactor with 2 temperature zones.

\section{CONCLUSION}

L'approche décrite dans ce travail est entièrement basée sur l'expérience. Il faut rappeler tout d'abord que les mesures expérimentales dans le volume de contrôle « réacteur » ne permettent que la détermination des relations fonctionnelles entre cinétiques de réaction et concentrations en phase gazeuse. En fait, seule cette phase gazeuse est accessible et nous ignorons ce qui se passe à la surface du solide.
Néanmoins, l'évolution du système réactionnel peut être quantifiée avec une bonne précision à l'aide de réseaux de réactions globales. Le choix du réseau, spécialement son unicité et sa complexité, dépend du but de l'étude et des informations disponibles. En pratique, le réseau le plus simple sera choisi. L'interprétation est toutefois basée sur l'hypothèse qu'il n'y a pas de transformation de phase du catalyseur solide le long du réacteur.

Le réseau et les valeurs des coefficients cinétiques dérivés permettent la définition de grandeurs caractéristiques du système. Ceci rend possible la comparaison des performances de catalyseurs, l'adaptation du mode opératoire du réacteur et par ce biais l'amélioration du rendement ainsi qu'un changement d'échelle fiable.

\section{REMERCIEMENTS}

Je tiens à remercier mes anciens thésards, Stefan Breiter, Marvin Estenfelder, André Quast et Klaus Wittstock pour la qualité de leur travail.

\section{RÉFÉRENCES}

1 Riekert, L. (1985) Observation and Quantification of Activity and Selectivity of Solid Catalysts. Appl. Catal., 15, 89-102.

2 Kotter, M. et Riekert, L. (1983) Beeinflussung der Aktivität und Selektivität von Tränkkatalysatoren durch die topographische Verteilung der Aktivkomponente. VT Verfahrenstechnik, 17, 639-658.

3 Breiter, S. (1995) Synergismus von oxidischen Katalysatorkomponenten bei der partiellen Oxidation von Isobuten zu Methacrolein. PhD Thesis, Universität Karlsruhe.

4 Kotter, M., Riekert, L. et Weyland, F. (1983) Deposition of Ternary Oxides as Active Compounds by Impregnation of Porous Carriers. In Preparation of Catalysts III, Poncelet, G., Grange, P. and Jacobs, P.A. (edts.), Elsevier, Amsterdam, 521-530

5 Recknagel, R. et Riekert, L. (1994) Kinetik der katalytischen Oxidation von Acrolein zu Acrylsäure. Chem. Technik, 46, 324-331

6 Breiter, S. et Lintz, H.G. (1995) Partial Oxidation of Isobutene to Methacrolein on $\mathrm{BiW} / \mathrm{FeCOMoK}$ Mixed Oxide Catalysts. Chem. Eng. Sci., 50, 785-791.

7 Lintz, H.G. et Wittstock, K. (1996) Catalytic Combustion of Solvent-Containing Air on Base Metal Catalysts. Catal. Today, 27, 237-242.

8 Quast, A. (1999) Partielle Oxidation von C4-Kohlenwasserstoffen zur Darstellung von Maleinsäureanhydrid und Furan. PhD Thesis, Universität Karlsruhe.

9 Lintz, H.G. et Quast, A. (1999) Partial Oxidation in the Integral Reactor: Possibilities of a Mathematical Description. Chem. Eng. Technol., 22, 817-822.

10 Becker, D., Kotter, M. et Riekert, L. (1989) Zur Selektivität der partiellen Oxidation von Propen an Schalenkatalysatoren. Chem. Ing. Tech., 61, 151-152.

11 Kotter, M., Li D.X. et Riekert, L. (1990) Partial Oxidation of o-Xylene to Phthalic Anhydride in a Structured Fixed Bed 
Containing a Sequence of Catalysts. Stud. Surf. Sci. Catal., 55, 267-274.

12 Kotter, M., Li. D.X. et Riekert, L. (1991) Partielle Oxidation von o-Xylol zu Phthalsäureanhydrid in einem strukturierten Festbettreaktor. DECHEMA-Monographie Katalyse, Kral, H. (ed.), Frankfurt/M., 122, 93-123.

13 Beschmann, K., Fuchs, S. et Hahn, T. (1997) Ammoxidation of Xylenes - Kinetics and Selectivity. 3rd World Congress on Oxidation Catalysis, Grasselli, R.K., Oyama, S.T., Gaffney, A.M. and Lyons, J.E. (eds.), 929-937.

14 Lintz, H.G. et Wittstock, K. (2001) The Oxidation of Solvents in Air on Oxidic Catalysts-Formation of Intermediates and Reaction Network. Appl. Catal. A: General, 216, 217-225.

15 Matros, Y., Noskov, A. et Chumachenko, V.A. (1993) Progress in Reverse-Process Application to Catalytic Incineration Problems. Chem. Eng. Proc., 32, 89-98.
16 Eigenberger, G. et Nieken, U. (1991) Katalytische Abluftreinigung: Verfahrenstechnische Aufgaben und neue Lösungen. Chem. Ing. Tech., 63, 781-791.

17 Fink, K., Gajewski, W., Joisten, M., Neufert, R., Sprehe, J. et Zürbig, J. (1992) Katalyse in der Abluftreinigung. Chem. Ing. Tech., 64, 416-421.

18 Kotter, M. (1992) Katalytische Abluftreinigung mit integriertem regenerativem Wärmetausch. Chem. Ing. Tech., 64, 848 .

19 Lintz, H.G. et Quast, A. (1997) Partial Oxidation of Butadiene to Furan at Bismuth Molybdate Catalysts. Catal. Letters, 46, 255-259.

20 Estenfelder, M., Lintz, H.G., Stein, B. et Gaube, J. (1998) Comparison of Kinetic Data Obtained from Integral Fixed Bed Reactor and Differential Recycle Reactor. Chem. Eng. Proc., 37, 109-114.

Manuscrit définitif reçu en août 2002 